\title{
Effect of perioperative amplitude-integrated electroencephalography on neurodevelopmental outcomes following infant heart surgery
}

\author{
JUAN GUI $^{1,2}$, SUIXIN LIANG ${ }^{2}$, YUNXIA SUN ${ }^{2}$, YUMEI LIU ${ }^{2}$, CHEN CHEN $^{2}$, \\ BI WANG ${ }^{2}$, JING ZHONG ${ }^{2}$, YUHUI YU ${ }^{2}$ and SHAORU HE $\mathrm{HE}^{1-3}$
}

${ }^{1}$ The Second School of Clinical Medicine, Southern Medical University, Guangzhou, Guangdong 510515;
${ }^{2}$ Department of NICU, Guangdong Provincial People's Hospital, Guangdong Academy of Medical Sciences;
${ }^{3}$ Department of Neonatal Cardiology, Guangdong Provincial Cardiovascular Institute, Guangzhou, Guangdong 510080, P.R. China

Received November 12, 2018; Accepted August 5, 2019

DOI: $10.3892 /$ etm.2020.9004

\begin{abstract}
The purpose of the current study was to determine the effect of perioperative amplitude-integrated electroencephalography (aEEG) on neurodevelopmental outcomes in infants with congenital heart disease (CHD). A total of 93 children with CHD were included in the current study. All patients enrolled in the present study had undergone cardiac surgery prior to 3 months of age and pre- or postoperative aEEG was monitored. Participants were assessed after 1 year using the Bayley Scales of Infant Test. A total of $82.2 \%$ of infants exhibited continuous normal voltage preoperatively (CNV) and $93.7 \%$ exhibited CNV postoperatively. Seizures were indicated in 2 infants preoperatively and 3 infants postoperatively. Compared with infants with PDI, infants with cyanotic CHD $(\beta=17.218)$ exhibited a significantly lower MDI, an increased length of intensive care stay, and lower PDI scores $(\beta=-0.577)$. Infants that underwent surgery with $\mathrm{CPB}$ exhibited higher PDI scores $(\beta=11.956)$. Infants that exhibited behavioral problems also had lower PDI scores $(\beta=-10.605)$. An abnormal preoperative background pattern and an absent postoperative SWC independently predicted poorer motor $(\mathrm{P}=0.014)$ and cognitive $(\mathrm{P}=0.049)$ outcomes at 1 year. The current study demonstrated that infants with CHD who underwent cardiac surgery prior to 3 months of age exhibited delayed neurodevelopmental
\end{abstract}

Correspondence to: $\mathrm{Dr}$ Shaoru He, Department of NICU, Guangdong Provincial People's Hospital, Guangdong Academy of Medical Sciences, 106 Zhongshan Er Road, Guangzhou, Guangdong 510080, P.R. China

E-mail: guijuan224@126.com

Abbreviations: aEEG, amplitude-integrated electroencephalography; $\mathrm{CHD}$, congenital heart disease; $\mathrm{CNV}$, continuous normal voltage; DNV, Discontinuous normal voltage; MDI, mental developmental index; PDI, psychomotor developmental index; SWC, sleep-wake cycle

Key words: brain injury, amplitude-integrated electroencephalography, heart surgery, neurodevelopmental outcomes outcomes, and that an aEEG assessment can aid in predicting these outcomes following surgery.

\section{Introduction}

Congenital heart disease (CHD) is the most common birth defect, affecting $\sim 0.8 \%$ of live births worldwide $(1,2)$. The true incidence of CHD in China was 11.1 per 1,000 live births, which is higher than previously reported (3). Due to the an increasing absolute and relative survival in children and young adults with CHD, brain injury, as a potentially devastating complication of CHD, has attracted increasing attention from neurologists and parents (4). With improved surgical outcomes and survival rates, children with CHD exhibit an increased risk of developing neurodevelopmental deficits, which are characterized by mild cognitive dysfunction, social interaction problems and difficulties in core communication (4). Cognitive and global motor developments of infants and children can be evaluated using the Bayley test, which a reliable and widely applied test for growth restriction in children aged 12 months (5). This test was first described in 1969 by Bayley (6), and revised and standardized for the Chinese population in 1993 (7).

Amplitude-integrated electroencephalography (aEEG) is used to identify seizures and abnormalities in the background cerebral activity of infants in Neonatal Intensive Care Units (NICU) (8). Continuous electroencephalography (EEG) provides an image of the brain's surface electrical activity and is a time-sensitive method of detecting brain injury. For continuous bedside monitoring and time-compressed background patterns, aEEG is a simpler and more convenient tool that is used in the NICU for the assessment of brain function in high-risk neonates, compared with a regular EEG (9). In hypoxic ischemic encephalopathy (HIE) and preterm infants, aEEG is helpful for predicting the short-term outcome (10), as a continuous bedside monitor that can indicate the abnormalities in cerebral activity immediately (11). Additionally, aEEG is a non-invasive and accurate approach that can assess neurodevelopmental maturation and brain injuries in premature infants, or in infants exhibiting cardiac arrest, extracorporeal 
membrane oxygenation (ECMO) and ligation of patent ductus arteriosus. A perioperative aEEG may be used as a tool to predict clinical outcomes in infants undergoing heart surgery for CHD (12). However, the role of aEEG in the prediction of the outcomes of patients with CHD, following surgery, remains largely undetermined. The current study aimed to determine the effect of perioperative aEEG on neurodevelopmental outcomes in infants with CHD.

\section{Patients and methods}

Patients. A retrospective cohort of 93 consecutively enrolled infants (50 males, 43 females), born with CHD were enrolled in the current study from Guangdong Provincial People's Hospital between September 2015 and January 2017. For infants $>3$ months of age, surgery is difficult and perioperative hemodynamics fluctuates greatly. Additionally, the immune system is not fully developed prior to 3 months of age. Therefore, infants are prone to infection following cardiac surgery. The brain of infants prior to the age of 3 months is vulnerable to ischemia and hypoxia. Therefore, children with CHD exhibit improved surgical outcomes, lower infection rates and higher survival rates within 3 months after birth. Infants with a genetic comorbidity or suspected syndromic disorder were excluded from the current study (13). All demographic, perioperative and cardiac measurements were collected retrospectively from each patient. The ethnicity of the patient's family and the family socioeconomic status were assessed. The current study was approved by the ethical committee of Guangdong Provincial People's Hospital, and written informed consent was obtained from all parents.

Grouping. Grouping patients by the type of CHD is generally accepted in the study of CHD development (12) or the analysis of the factors influencing neurodevelopment, patients were classified according to surgical factors, including surgery with or without orthopedic surgery and palliative surgery, immediate thoracic closure following surgery and delayed thoracic closure following surgery, which is not a common grouping used in other randomized controlled trial studies (grouping was based on disease classification and surgical details).

Clinical characteristics. Patient data included gestational age, birth weight, Apgar scores (14), cardiovascular function, respiratory and multi-organ failure, neurologic examination and neuroimaging data. The following information was recorded in the current study: Period and method of surgery, cardiopulmonary bypass time and aortic cross-clamping time. Simple and complex CHD was categorized depending on Risk Adjustment in Congenital Heart Surgery-1 $(15,16)$, which determined category 1 as simple CHD.

aEEG measurements. aEEG was monitored for $24 \mathrm{~h}$ prior to cardiac surgery using an 8-channel EEG (NicoletOne monitor; CareFusion Corp; Becton-Dickinson and Company). A total of 8 disposable, self-adhesive, EEG scalp electrodes (Blue Sensor BRS-50 K AmbuTM ECG electrode; Medicotest A/S) were used in a reduced montage following the international 10-20 system (17). The clinician that performed the main offline aEEG analyses was not involved in clinical care of the patients. The 8-channel cross-brain aEEG trace was derived and displayed at $6 \mathrm{~cm} / \mathrm{h}$ on paper using a semi logarithmic scale to assess and classify the aEEG background pattern. The channels were also used to record EEG data to describe episodes of EEG seizures in $10 \mathrm{sec}$ epochs.

The interpretation of aEEG. aEEG is commonly used to evaluate background pattern, seizure activity and sleep-wake cycles (18). aEEG traces were classified using background voltage (19) and a descriptive pattern. The aEEG recordings were categorized as 5 background patterns which were continuous normal voltage $(\mathrm{CNV})$, discontinuous normal voltage (DNV), or a combined third group of the severe aEEG voltage pattern (SEVP), including burst suppression (BS), continuous low voltage (CLV) or a flat trace (FT). For outcome analysis, the 5 background patterns was categorized into normal (CNV), mild abnormal (DNV transient and persistent) and severely abnormal (BS, CLV and FT) (20). Sleep-wake cycling (SWC) refers to the cyclic fluctuation in the amplitude and degree of discontinuity as the neonate enters various stages of sleep or wakefulness (21). On the aEEG, SWC is reflected by the presence of smooth sinusoidal variation, mostly in the minimal amplitude. Periods of wider bandwidth represent discontinuous activity during quiet sleep, whereas periods with a narrow bandwidth represent more continuous activity when awake or during active sleep (18). SWC was categorized by occurrence: Absent, immature or developed (18). An electrographic seizure was defined as an evolving repetitive stereotyped waveform, with a definite onset, a peak and an end that lasted for $\geq 10 \mathrm{sec}$ on raw EEG data (18). Antiepileptic drugs (Midazolam and Phenobarbital) were used to treat clinical seizures. The results of continuous aEEG/EEG monitoring were examined following patient discharge, by an external collaborator. Electrographic seizure activity (EA) was divided into the following groups: i) No seizure; ii) a single seizure (SS), in which the amplitude of a single waveform appeared suddenly and showed persistent cerebral cortex activity; iii) recurrent seizure (RS), in which a recurring amplitude indicated sudden and persistent cerebral cortex activity. All patient reports were examined by a neonatal neurological expert who was blinded from the patients' diagnosis.

Surgical management. Cardiac surgery was performed by one group of cardiac surgeons. The alphastat blood gas management was routinely performed. In the majority of cardiac surgeries, normothermic or mild hypothermic cardiopulmonary bypass (rectal temperature $>32^{\circ} \mathrm{C}$ or $28-32^{\circ} \mathrm{C}$, respectively) was performed with a pump flow rate of $100-150 \mathrm{ml} / \mathrm{kg} / \mathrm{min}$ to achieve a mean arterial pressure of $40-50 \mathrm{mmHg}(22,23)$. During arch repair, antegrade cerebral perfusion, with a pump flow rate of $-50 \mathrm{ml} / \mathrm{kg} / \mathrm{min}$ was used, and the mean arterial pressures were targeted at $-40-50 \mathrm{~mm} \mathrm{Hg}$ and were measured in the right radial artery. Modified ultra-filtrations were performed in all patients. Following surgery, patients received milrinon $(0.5-1 \mu \mathrm{g} / \mathrm{kg} / \mathrm{min})$ as an early postoperative treatment combined with noradrenaline $(0.05-1 \mu \mathrm{g} / \mathrm{kg} / \mathrm{min})$ and $/$ or adrenaline $(0.01-0.5 \mu \mathrm{g} / \mathrm{kg} / \mathrm{min})$ to treat cardiac failure. Postoperative analgesia included fentanyl (1-3 $\mu \mathrm{g} / \mathrm{kg} / \mathrm{h}$ within 3 days after surgery). Patients were given a Paracetamol drip, which exhibits a smaller 
influence on the aEEG compared with intravenous sedative/pain medications, to control pain when patients did not require ventilation.

Follow-up. All 93 infants received a follow-up (at 6 months, 1 year, 1 a half years and 2 years) at the Child Development Clinic of the Guangdong Provincial People's Hospital. At 1 year of age, the Bayley Scales of Infant Development was used to provide a mental development index (MDI) and psychomotor developmental index (PDI). Cognitive and global motor development were evaluated using the Bayley test, as previously described (6). The Bayley composite test scores are scaled over a range of 50-150, with a mean of 100 and a standard deviation (SD) of 16 (7). Although not considered to be an intelligence quotient test, the Bayley test reliably identifies infants with developmental delays, as indicated by scores of $<68$ (mean-2SD) on the cognitive or the global motor composite scores. Tests were performed by one clinician who was experienced in testing child development and was blinded to the patients' diagnosis and surgical details.

Statistical analysis. Data were processed using SPSS 16.0 (SPSS, Inc.) software and presented as the mean \pm SD. Comparisons between groups were performed using a student t-test and Bonferroni correction. Variance analysis or a signed-rank test was used for comparison of continuous variables and a $\chi^{2}$ test or Fisher's exact test for dichotomous variables. Comparisons with the grade data were performed using a Wilcoxon signed-rank test. Multiple linear regression analysis was used to determine the influencing factors of MDI and PDI. The $\beta$ value or the regression coefficient, was applied for regression analysis. $\beta>0$, indicated a positive association between strain and independent variable, and $\beta<0$, indicated a negative association. The inspection standard was bilateral 0.05 . $\mathrm{P}<0.05$ was considered to indicate a statistically significant difference.

\section{Results}

Clinical characteristics of infants. The characteristics of patients are presented in Table I. A total of 16 infants were born at $<37$ weeks of gestation (27-36.6 weeks) and surgery in these children was performed at term equivalent ( $>37$ weeks of gestation). Cardiac diagnoses were divided into four diagnostic groups according to Clancy et al (24) (Table II). A total of 5 children had $>1$ bypass surgery by the age of 1 years old.

aEEG characteristics of the infants with CHD preoperatively and postoperatively. A total of 45 infants were monitored using aEEG preoperatively and 79 were monitored postoperatively. A total of 31 infants underwent preoperative and postoperative aEEG. The aEEG results are presented in Table III. Preoperatively, $82.2 \%$ infants indicated CNV $(n=37)$ and $17.8 \%$ infants indicated DNV $(n=8)$. A total of 2 infants exhibited seizure activity on the aEEG. A total of 3 infants with preoperative SS were found without postoperative seizure. Postoperatively, discontinuous normal voltage was detected in $5(6.3 \%)$ infants, and continuous normal voltage was detected in $74(93.7 \%)$ infants. A total of 3 infants exhibited seizure activity on the postoperative aEEG without seizure activity on the preoperative aEEG. One of the 3 infants
Table I. Patient characteristics $(n=93)$.

\begin{tabular}{lc}
\hline Characteristics & Value \\
\hline Male sex, n (\%) & $50(53.8 \%)$ \\
Gestational age (weeks) & $38.18 \pm 2.799$ \\
Birth weight (g) & $2779.60 \pm 662.650$ \\
Cyanotic CHD, n (\%) & $46(49.5)$ \\
Complex CHD, n (\%) & $58(62.4)$ \\
Age at surgery (months) & $1.56 \pm 1.373$ \\
Duration of intensive care stay (day) & $27.31 \pm 12.319$ \\
Emergency operation (\%) & $14(15.05)$ \\
Corrective surgery (\%) & $88(94.62)$ \\
CPB (min) & $114.11 \pm 68.545$ \\
Aortic clamp time (min) & $66.949 \pm 42.759$ \\
Delayed sternal closured (\%) & $27(29.03)$ \\
Mechanical ventilation (day) & $8.022 \pm 8.106$ \\
\hline
\end{tabular}

CHD, congenital heart disease; CPB, cardiopulmonary bypass; d, days.

also had symptomatic seizures and was treated with phenobarbital $(10 \mathrm{mg} / \mathrm{kg} /$ day $)$ and the magnetic resonance imaging was normal (Table III).

MDI and PDI in infants with CHD. All 93 infants were examined at the mean age of 10.54 months. The mean MDI was $96.483 \pm 21.997$, and PDI was $87.763 \pm 21.584$ (data not shown). PDI was significantly lower than the normal values of 100 $(\mathrm{P}<0.001)$, with no significant differences observed for MDI $(\mathrm{P}=0.127$ ) (Table IV). The mean of MDI and PDI in cyanotic CHD was compared with acyanotic CHD (MDI, 93.00 \pm 21.422 vs. 99.89 \pm 22.244 ; PDI, 84.50 \pm 20.397 vs. $90.96 \pm 22.443$, data not shown). The mean of MDI and PDI was lower compared with complex and simple CHD (MDI,94.45 \pm 20.649 vs.99.86 \pm 23.993 ; PDI, $86.81 \pm 19.617$ vs. $89.34 \pm 24.725$, data not shown). However, the differences were not statistically significant.

Risk factors for abnormal MDI and PDI. According to univariate linear regression and multivariate linear regression, the results demonstrated a positive linear association between PDI and MDI ( $\beta=0.929$; 95\% CI: Lower, 0.556; upper, 1.301; Fig. 1; Table V). A negative linear association between length of intensive care stay and PDI was observed ( $\beta=-0.577$; 95\% CI: Lower, -0.874; upper, -0.279 ; Table VI), and CPB was the protective factor of PDI $(\beta=11.956 ; 95 \% \mathrm{CI}$ : Lower, 0.845; upper, 23.068; Table VI). The PDI of surgery with $\mathrm{CPB}$ was significantly higher than that of surgery without $\mathrm{CPB}(\mathrm{P}=0.035$; data not shown). The PDI of infants with mild behavioral problems was significantly lower compared with infants exhibiting no behavioral problems $(\beta=-10.605 ; 95 \% \mathrm{CI}$ : Lower, -18.546; upper, -2.664; $\mathrm{P}=0.01$; Table VI).

Association between perioperative aEEG and neurodevelopmental outcomes. The association between perioperative aEEG and neurodevelopmental outcomes was subsequently examined. A significant difference of MDI was demonstrated among groups with developed, immature or absent 
Table II. Cardiac diagnoses and cardiac class.

\begin{tabular}{lccccc}
\hline Cardiac diagnoses & Overall $(\mathrm{n}=93)$ & Class I $(\mathrm{n}=68)$ & Class II $(\mathrm{n}=10)$ & Class III $(\mathrm{n}=13)$ & Class IV $(\mathrm{n}=2)$ \\
\hline TGA/IVS & 8 & 8 & 0 & 0 & 0 \\
TGA/VSD & 12 & 12 & 0 & 0 & 0 \\
TOF & 1 & 1 & 0 & 1 & 0 \\
TOF/PA & 1 & 0 & 0 & 0 & 0 \\
TAPVC & 9 & 9 & 0 & 0 & 0 \\
VSD without IAA/coarctation & 30 & 30 & 10 & 0 & 0 \\
VSD with IAA/coarctation & 10 & 0 & 0 & 0 & 0 \\
AVC defect & 0 & 0 & 0 & 0 & 12 \\
HLHS & 1 & 0 & 0 & 0 & 1 \\
Other functional single ventricle & 13 & 0 & 0 & 0
\end{tabular}

The congenital heart defects of the infants were classified into 1 of 4 categories based on preoperative echocardiograms: class I, two-ventricle heart without arch obstruction; class II, two-ventricle heart with arch obstruction; class III, single ventricle heart without arch obstruction, and class IV, single ventricle heart with arch obstruction. TGA, transposition of the great arteries; IVS, intact ventricular septum; VSD, ventricular septal defect; TOF, tetralogy of Fallot; PA, pulmonary artery; TAPVC, total anomalous pulmonary venous connection; IAA, interrupted aortic arch; AVC, atrioventricular canal; HLHS, hypoplastic left heart syndrome.

Table III. Preoperative and postoperative aEEG characteristics of infants with CHD ( $n=93)$.

\begin{tabular}{lcc}
\hline Characteristics & $\begin{array}{c}\text { Preoperative } \\
(\mathrm{n}=45)\end{array}$ & $\begin{array}{c}\text { Postoperative } \\
(\mathrm{n}=79)\end{array}$ \\
\hline Background n (\%) & $37(82.2)$ & $74(93.7)$ \\
Normal & $8(17.8)$ & $5(6.3)$ \\
Mild abnormal & $0(0)$ & $0(0)$ \\
Severe abnormal & & \\
SWC n $(\%)$ & $9(20)$ & $35(44.3)$ \\
Developed & $34(75.6)$ & $41(51.9)$ \\
Immature & $2(4.4)$ & $3(3.8)$ \\
Absent & & $76(96.2)$ \\
Seizure n $(\%)$ & $43(95.6)$ & $2(2.5)$ \\
No & $1(2.2)$ & $1(1.3)$ \\
SS & $1(2.2)$ & \\
RS & & \\
\hline
\end{tabular}

aEEG, amplitude-integrated electroencephalography; CHD, congenital heart disease; normal, continuous normal voltage; mild abnormal, discontinuous normal voltagetransient and persistent; severe abnormal, burst suppression, continuous normal voltage and flat trace; SS, single seizure; RS, recurrent seizure.

postoperative SWC ( $\mathrm{t}=3.984 ; \mathrm{P}=0.023$; Table VII). Using the Bonferroni method for further comparisons, infant MDI, with absent postoperative SWC $(70.00 \pm 34.641)$ was indicated to be significantly lower compared with immature postoperative SWC MDI (100.65 \pm 19.572 ; $\mathrm{P}<0.001$; Fig. 2). Additionally, infants with mild abnormal exhibited a significantly lower PDI compared with CNV ( $\mathrm{t}=2.87$; $\mathrm{P}=0.0064$; Table VIII; Fig. 3). A single infant with preoperative SS without postoperative
EA exhibited a poor outcome at 1 year (MDI 80, PDI 84, data not shown). A total of 2 infants with postoperative SS also exhibited a poor outcome (MDI 79, PDI 67; MDI 97, PDI 116, respectively, data not shown). These three infants both included in the cyanotic CHD group. A single infant with postoperative RS and symptomatic seizure with VSD exhibited lower PDI but normal MDI $(94,110$, respectively, data not shown). Due to the small number of patients which indicated seizure activity pre- or postoperation (only 4 children), a significant difference was not revealed between seizure and clinical characteristics. These data were not shown. A close association was indicated between perioperative aEEG and neurodevelopmental outcomes.

Predictive value of the perioperative aEEG on neurodevelopmental outcomes. The predictive value of the perioperative aEEG results on neurodevelopmental outcomes was assessed in 31 infants that underwent pre- and postoperative aEEG using Wilcoxon signed-rank test. The comparison of pre- and postoperative aEEG suggested that infant brain function exhibited no changes following surgery (Table IX). The PDI $(68.43 \pm 19.217)$ of infants with mild abnormal preoperative background pattern was significantly lower compared with normal preoperative background pattern (92.97 \pm 21.005 ; $\mathrm{P}=0.013$; Table VIII; Fig. 4). PDI $(85.08 \pm 21.431)$ of infants with immature preoperative SWC was significantly lower compared with normal preoperative SWC (105.14 \pm 21.162 ; $\mathrm{P}=0.037$; $\mathrm{P}<0.05$; Table VIII; Fig. 4). These results suggested that the perioperative aEEG may serve as a predictor of neurodevelopmental outcomes.

\section{Discussion}

The aim of the current study was to determine the predictive value of pre- and postoperative aEEG on the 1-year neurodevelopmental outcome in children who underwent a CHD operation. 
Table IV. MDI and PDI in infants with CHD and the average of the normal population (100). The BSID (Chinese City) was standardized in 15 cities and defined normal value in general population was $100(9)$.

$95 \%$ CI of the difference

\begin{tabular}{lccccrr} 
& $\mathrm{t}$ & $\mathrm{df}$ & P-value & Mean difference & Lower & Upper \\
\hline MDI & -1.542 & 92 & 0.127 & -3.51613 & -8.0463 & 1.0141 \\
PDI & -5.467 & 92 & $<0.001^{\mathrm{a}}$ & -12.23656 & -16.6818 & -7.7913 \\
\hline
\end{tabular}

${ }^{\mathrm{a}} \mathrm{P}<0.05$. MDI, Mental developmental index; PDI, Psychomotor developmental index; CHD, Congenital heart disease; CI, confidence interval.

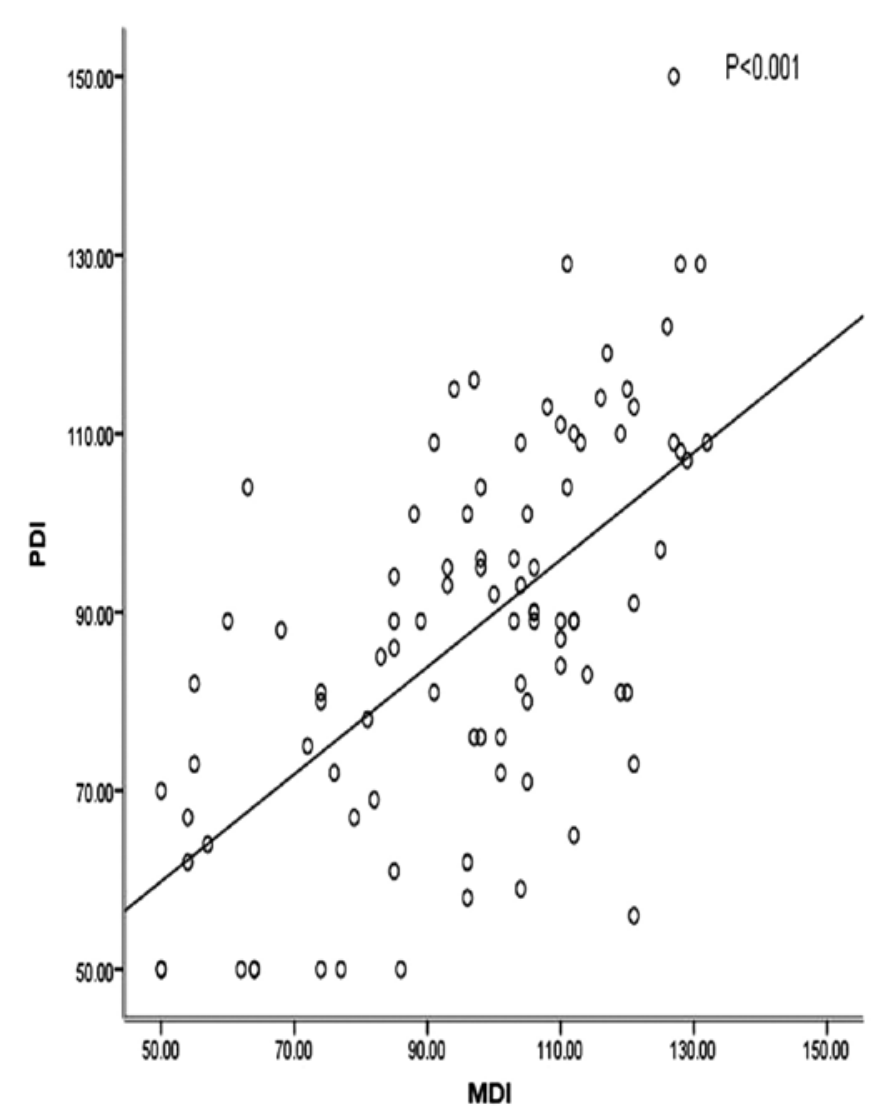

Figure 1. MDI and PDI of infants. Association of MDI and PDI based on univariate linear regression and multivariate linear regression. MDI, mental developmental index; PDI, psychomotor developmental index.

This information is important as imaging studies fail to indicate a relationship between preoperative cranial ultrasound findings and neurodevelopmental outcomes, or between perioperative brain injuries detected on cerebral magnetic resonance (25). In the current study, the perioperative aEEG was strongly associated with neurodevelopmental outcomes at 1 year of age and was independent of confounding factors. The results of the current study extend and support the findings of a previous study conducted by Gunn et al (26), which demonstrated similar background pattern changes in relation to surgery, and indicated an association between postoperative background pattern abnormalities and a poorer neurodevelopmental outcomes at 2 years of age.

The majority of infants in the current study (80.6\%) exhibited a normal background pattern, with $19.4 \%$ exhibiting DNV. An increasing number of infants with cyanotic CHD
Table V. Multivariate linear regression analysis of the influencing factors of MDI.

\begin{tabular}{lrrrrrr}
\hline & & & & \multicolumn{2}{c}{$\begin{array}{l}95 \% \text { CI of } \\
\text { regression } \\
\text { coefficient }\end{array}$} \\
\cline { 5 - 7 } $\begin{array}{l}\text { Influencing } \\
\text { factor }\end{array}$ & $\beta$ & $\mathrm{t}$ & P-value & Lower & Upper \\
\hline PDI & 0.929 & 5.261 & $<0.001^{\mathrm{a}}$ & 0.556 & 1.301 \\
Cyanotic & 17.218 & 2.385 & $0.029^{\mathrm{a}}$ & 1.984 & 32.451 \\
CHD & & & & & & \\
\hline
\end{tabular}

${ }^{\mathrm{a}} \mathrm{P}<0.05$. MDI, mental developmental index; PDI, psychomotor developmental index; CHD, congenital heart disease; CI, confidence interval.

or complex CHD exhibited significantly abnormal preoperative SWC, which was contradictory to a study performed by ter Horst et al (20) that demonstrated that SWC was equal between infants with cyanotic and acyanotic CHD.

A total of $6.3 \%$ of infants exhibited an abnormal background pattern. The percentage of DNV was smaller in postoperative aEEG compared with preoperative aEEG, indicating that brain function of infants with CHD can be improved following surgery. This is consistent with the results of a previous study on perioperative aEEG in 73 infants with CHD. The reason for the smaller number of DNV was the improvement of oxygen delivery and cerebral perfusion following surgery. The improvement is consistent with the study by Anastasia Dimitropoulos which indicated that a postoperative acquired brain injury was less frequent than preoperative injury $(41 \%$ preoperative vs. $30 \%$ postoperative) (27). However, the improvement of cerebral activity was not observed in 31 infants who underwent both pre- and post-operative aEEG. Another study (28) reported that MRI-defined white matter injuries before and after surgery were similar. A proportion of infants in the present study, who were transferred from a basic hospital, usually exhibited unfavorable situations prior to surgery and also may have worse brain function. Therefore, a large cohort clinical study is required to confirm this finding.

EA was present in $2(4.4 \%)$ infants preoperatively and in $3(3.8 \%)$ infants postoperatively. Three infants exhibited cyanotic CHD, which is consistent with the study of Gunn et al (26), demonstrating that perioperative seizures 
Table VI. Multivariate linear regression analysis of influencing factors of PDI.

$95 \% \mathrm{CI}$ of

regression coefficient

\begin{tabular}{lcccrr}
\cline { 4 - 5 } Influencing factor & $\beta$ & $\mathrm{t}$ & P-value & Lower & Upper \\
\hline MDI & 0.497 & 6.397 & $<0.001^{\mathrm{a}}$ & 0.342 & 0.652 \\
Length of ICU & -0.577 & -3.861 & $<0.001^{\mathrm{a}}$ & -0.874 & -0.279 \\
Surgery with CPB & 11.956 & 2.142 & $0.035^{\mathrm{a}}$ & 0.845 & 23.068 \\
Mild behavioral problem & -10.605 & -2.658 & $0.01^{\mathrm{a}}$ & -18.546 & -2.664 \\
Severe behavioral problem & -4.784 & -0.645 & 0.521 & -19.54 & 9.973
\end{tabular}

${ }^{\mathrm{a}} \mathrm{P}<0.05$. PDI, Psychomotor developmental index; CPB, cardiopulmonary bypass; CI, confidence interval.

Table VII. Association of MDI and perioperative aEEG.

\begin{tabular}{lccc}
\hline aEEG characteristics & MDI (mean \pm SD) & F/t & P-value \\
\hline Preoperative background & & 1.96 & 0.0561 \\
Normal & $99.19 \pm 22.552$ & & \\
Mild abnormal & $80.57 \pm 25.448$ & & \\
Preoperative SWC & & 0.555 & 0.578 \\
Developed SWC & $102.22 \pm 25.680$ & & \\
Immature SWC & $94.50 \pm 23.685$ & & \\
Absent SWC & $106.50 \pm 7.778$ & & \\
Postoperative background & & 1.01 & 0.3139 \\
Normal & $95.05 \pm 21.605$ & & \\
Mild abnormal & $104.20 \pm 23.658$ & & \\
Postoperative SWC & & 3.984 & $0.023^{\mathrm{a}}$ \\
Developed SWC & $91.94 \pm 21.349$ & & \\
Immature SWC & $100.65 \pm 19.572$ & & \\
Absent SWC & $70.00 \pm 34.641$ & & \\
\hline
\end{tabular}

${ }^{\mathrm{a} P}<0.05$. MDI, Mental developmental index; aEEG, amplitude-integrated electroencephalography; SD, standard deviation; SWC, sleep-wake cycle.

are common in infants undergoing Norwood operations. Additionally, these findings are also in accordance with the study of Dimitropoulos et al (27), which indicated that lower preoperative oxygen saturation is a risk factor for brain injury. However, EA was demonstrated to be more frequent in infants with acyanotic CHD (20).

The results of the current study indicated that poorer PDI at 1 year was associated with an abnormal preoperative aEEG background pattern and SWC, and the same association was observed between poorer MDI at 1 year and abnormal postoperative SWC. This finding is consistent with the study by Gunn et al (12), in which an abnormal background pattern was associated with poorer neurodevelopmental outcome at 2 years of age. The significance of the DNV background pattern, as a potential negative outcome predictor, has been emphasized by Mulkey et al (29), in which DNV was considered to be mildly abnormal. Gunn et al (12) and Latal et al (30)

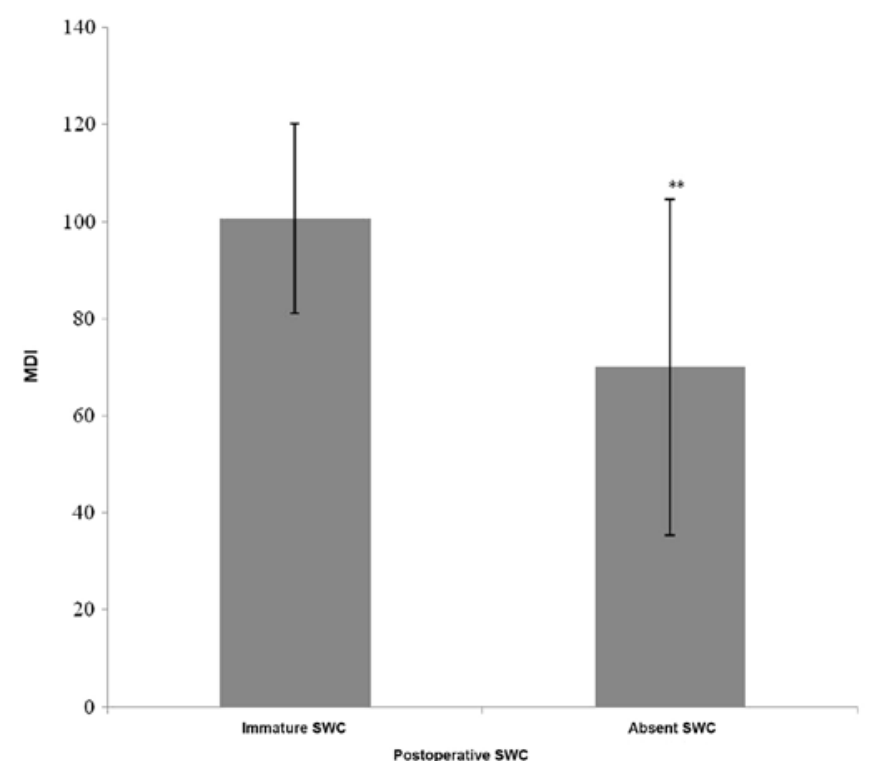

Figure 2. Comparison of the MDI in infants with absent and immature postoperative SWC using the Bonferroni method. ${ }^{* *} \mathrm{P}<0.01$. MDI, mental developmental index; SWC, sleep-wake cycle.

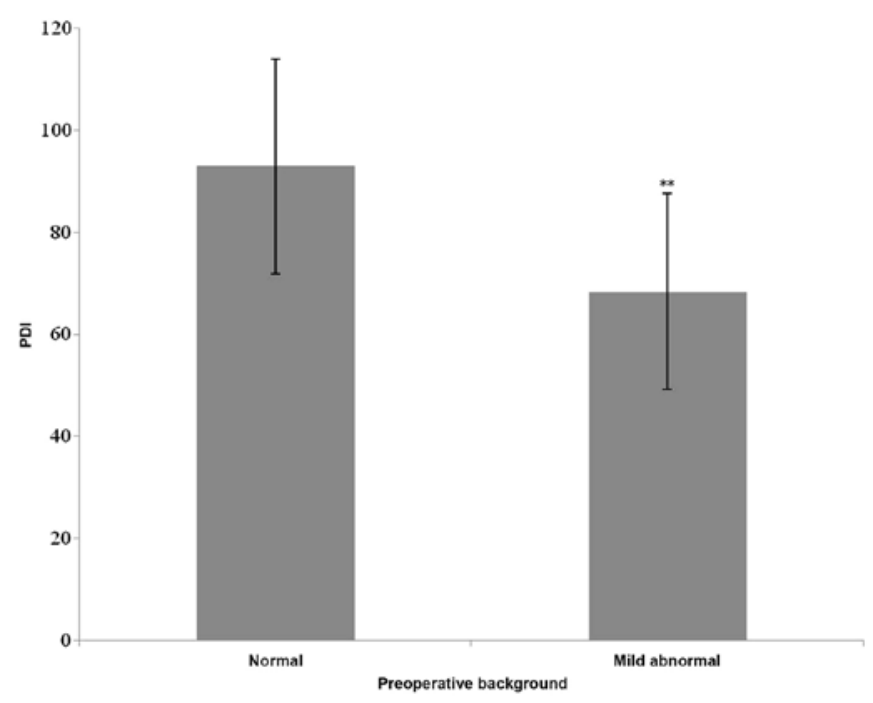

Figure 3. Comparison of the PDI in infants with normal and mild abnormal preoperative background pattern. ${ }^{* *} \mathrm{P}<0.01$. PDI, psychomotor developmental index. 
Table VIII. Association of PDI and perioperative aEEG.

\begin{tabular}{lccc}
\hline aEEG characteristic & PDI (mean \pm SD) & F/t & P-value \\
\hline Preoperative background & & 2.87 & $0.0064^{\mathrm{a}}$ \\
$\quad$ Normal & $92.97 \pm 21.005$ & & \\
Mild abnormal & $68.43 \pm 19.217$ & & \\
Preoperative SWC & & 2.18 & 0.126 \\
Developed SWC & $102.44 \pm 23.975$ & & \\
Immature SWC & $85.91 \pm 21.268$ & & \\
Absent SWC & $82.50 \pm 9.192$ & & \\
Postoperative background & & -0.95 & 0.3435 \\
Normal & $88.30 \pm 21.806$ & & \\
Mild abnormal & $79.60 \pm 20.070$ & & \\
Postoperative SWC & & 2.936 & 0.059 \\
Developed SWC & $83.86 \pm 21.756$ & & \\
Immature SWC & $92.51 \pm 20.839$ & & \\
Absent SWC & $68.00 \pm 17.088$ & & \\
\end{tabular}

${ }^{\mathrm{a}} \mathrm{P}<0.05$. PDI, Psychomotor developmental index; aEEG, amplitude-integrated electroencephalography; SWC, sleep-wake cycle; $\mathrm{SD}$, standard deviation.

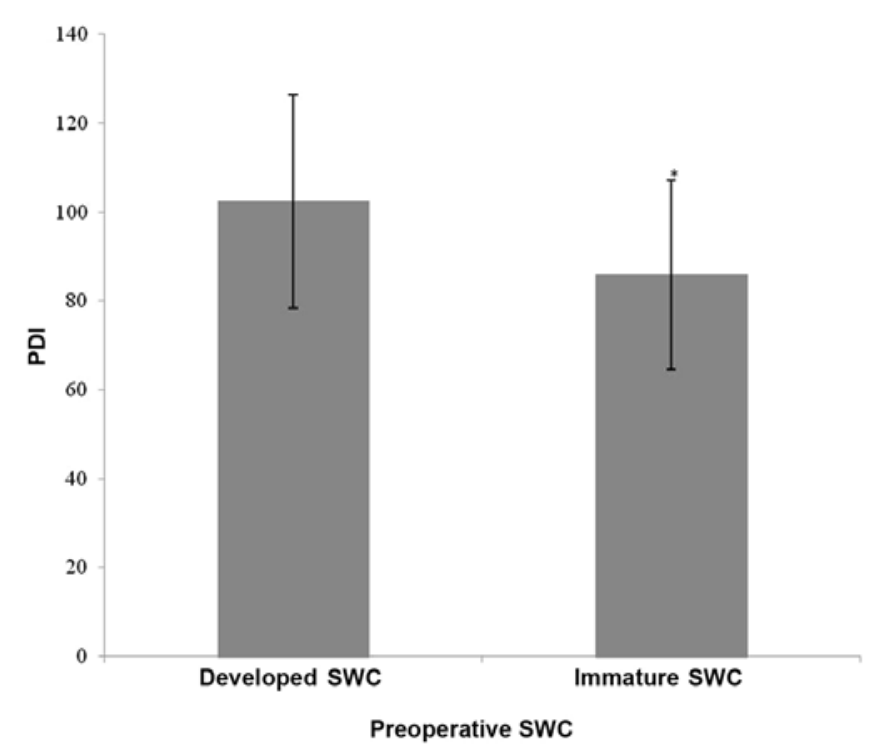

Figure 4. Comparison of the PDI in infants with developed and immature preoperative SWC background patterns. ${ }^{*} \mathrm{P}<0.05$. PDI, psychomotor developmental index; SWC, sleep-wake cycle.

demonstrated that a delayed recovery of SWC was associated with a poorer neurodevelopmental outcome in children with CHD. Considering the effect of sedative and pain relief drugs is difficult to avoid, the current study did not collect an intraoperative aEEG measurement. Therefore, preoperative aEEG may be a marker for a more complicated and severe clinical course and may independently predict neurodevelopmental outcome.

A total of 3.1 and $5.7 \%$ of all infants with proven pre- and postoperative seizure activity were indicated on the aEEG, respectively, and perioperative seizures may easily occur in
Table IX. Changes between pre- and postoperative aEEG $(\mathrm{n}=31)$.

\begin{tabular}{lrcr}
\hline Perioperative aEEG & $\mathrm{n}$ & $\mathrm{Z}$ & P-value \\
\hline $\begin{array}{l}\text { Background pattern } \\
\text { Better }\end{array}$ & 4 & 0.378 & 0.705 \\
$\quad$ No change & 24 & & \\
$\quad$ Worse & 3 & & \\
SWC & & 1.508 & 0.132 \\
$\quad$ Better & 8 & & \\
$\quad$ No change & 20 & & \\
$\quad$ Worse & 3 & & \\
Seizure & & 0 & 1.000 \\
$\quad$ Better & 1 & & \\
$\quad$ No change & 29 & & \\
$\quad$ Worse & 1 & & \\
\hline
\end{tabular}

aEEG, amplitude-integrated electroencephalography; SWC, sleep-wake cycle.

cyanotic CHD patients. These results were lower compared with other studies, which reported a postoperative prevalence of 11-19\% using either continuous electroencephalography recordings or aEEG (31). However, this result is similar to a previous study indicating a number of $6.7 \%$ (32). Accordingly, the prevalence of postoperative seizures was higher in a group of neonates following surgical correction of a congenital aortic arch obstruction (33). Therefore, the differences in the prevalence of aEEG seizures may be explained by the time of aEEG monitoring.

Postoperative seizures are a risk factor for poor neurodevelopmental outcomes in newborns with transposition of the great arteries (28) and for other types of CHD (34). Algra et al (31) demonstrated that postoperative seizures on aEEG were associated with new cerebral injuries and worsening motor outcomes at 2 years of age. In the present study, a weak association was indicated between perioperative seizures and poorer motor outcome at 1 year of age, which was consistent with two patient cohorts in which perioperative seizures were not associated with early neurodevelopmental outcome (35).

In the current study, a pre- and postoperative aEEG was not performed in all participants, as the patients may have been in the different department prior to surgery. Because only patients were in a severe situation before surgery were managed in NICU and could undergo both pre- and postoperative aEEG. Additionally, molecular level changes pre- and postoperative aEEG were not assessed in the current study, and this should be investigated in future research.

In conclusion, children with CHD who underwent cardiac surgery before 3 months of age exhibited delayed neurodevelopmental outcomes, and aEEG is a useful tool for predicting the neurodevelopmental outcomes in infants undergoing heart surgery.

\section{Acknowledgements}

Not applicable. 


\section{Funding}

No funding was received.

\section{Availability of data and materials}

The datasets used and/or analyzed during the current study are available from the corresponding author on reasonable request.

\section{Authors' contributions}

JG designed the study and wrote the manuscript. SL and YS collected the data from the patients. YL,CC and BW performed the experiment. JZ and YY analyzed the data. As the corresponding author of the article, SH performed the literature research, revised the manuscript and gave final approval of the version to be published. All authors read and approved the final manuscript.

\section{Ethics approval and consent to participate}

The current study was approved by the ethical committee of Guangdong Provincial People's Hospital, and written informed consent was obtained from all parents of the patients.

\section{Patient consent for publication}

Not applicable.

\section{Competing interests}

The author declare that they have no competing interest.

\section{References}

1. Mandalenakis Z, Rosengren A, Skoglund K, Lappas G, Eriksson $\mathrm{P}$ and Dellborg M: Survivorship in children and young adults with congenital heart disease in Sweden. JAMA Intern Med 177: 224-230, 2017.

2. Matthiesen NB, Henriksen TB, Gaynor JW, Agergaard P, Bach CC, Hjortdal VE and Østergaard JR: Congenital heart defects and indices of fetal cerebral growth in a nationwide cohort of 924422 liveborn infants. Circulation 133: 566-575, 2016

3. Qu Y, Liu X, Zhuang J, Chen G, Mai J, Guo X, Ou Y, Chen J, Gong W, Gao X, et al: Incidence of congenital heart disease: The 9-year experience of the Guangdong registry of congenital heart disease, China. PLoS One 11: e0159257, 2016.

4. Massaro AN, El-Dib M, Glass P and Aly H: Factors associated with adverse neurodevelopmental outcomes in infants with congenital heart disease. Brain Dev 30: 437-446, 2008.

5. Komur M, Ozen S, Okuyaz C, Makharoblidze K and Erdogan S: Neurodevelopment evaluation in children with congenital hypothyroidism by Bayley-III. Brain Dev 35: 392-397, 2013.

6. Kono Y, Yonemoto N, Kusuda S, Hirano S, Iwata O, Tanaka K and Nakazawa J: Developmental assessment of VLBW infants at 18 months of age: A comparison study between KSPD and Bayley III. Brain Dev 38: 377-385, 2016.

7. Luo XR, Yang ZW and Wan GB; National Collaboration Group: Revision of bailey infant and child development scale in China (City Edition). Chin J Clin Psychol 1: 71-75, 1993.

8. Massaro AN, Tsuchida T, Kadom N, El-Dib M, Glass P, Baumgart $\mathrm{S}$ and Chang T: aEEG evolution during therapeutic hypothermia and prediction of NICU outcome in encephalopathic neonates. Neonatology 102: 197-202, 2012.

9. Davis AS, Gantz MG, Do B, Shankaran S, Hamrick SE, Kennedy KA, Tyson JE, Chalak LF, Laptook AR, Goldstein RF, et al: Serial aEEG recordings in a cohort of extremely preterm infants: Feasibility and safety. J Perinatol 35: 373-378, 2015.
10. Fogtmann EP, Plomgaard AM, Greisen G and Gluud C: Prognostic accuracy of electroencephalograms in preterm infants: A systematic review. Pediatrics 139: e20161951, 2017.

11. Rakshasbhuvankar A, Paul S, Nagarajan L, Ghosh S and Rao S: Amplitude-integrated EEG for detection of neonatal seizures: A systematic review. Seizure 33: 90-98, 2015.

12. Gunn JK, Beca J, Hunt RW, Olischar M and Shekerdemian LS: Perioperative amplitude-integrated EEG and neurodevelopment in infants with congenital heart disease. Intensive Care Med 38: 1539-1547, 2012.

13. Homsy J, Zaidi S, Shen Y, Ware JS, Samocha KE, Karczewski KJ, DePalma SR, McKean D, Wakimoto H, Gorham J, et al: De novo mutations in congenital heart disease with neurodevelopmental and other congenital anomalies. Science 350: 1262-1266, 2015.

14. Iliodromiti S, Mackay DF, Smith GC, Pell JP and Nelson SM: Apgar score and the risk of cause-specific infant mortality: A population-based cohort study. Lancet 384: 1749-1755, 2014.

15. O'Brien SM, Clarke DR, Jacobs JP, Jacobs ML, Lacour-Gayet FG, Pizarro C, Welke KF, Maruszewski B, Tobota Z, Miller WJ, et al: An empirically based tool for analyzing mortality associated with congenital heart surgery. J Thorac Cardiovasc Surg 138: 1139-1153, 2009.

16. Lacour-GayetF,Clarke D,Jacobs J,Comas J,Daebritz S, Daenen W, Gaynor W, Hamilton L, Jacobs M, Maruszsewski B, et al: The Aristotle score: A complexity-adjusted method to evaluate surgical results. Eur J Cardiothorac Surg 25: 911-924, 2004.

17. Tekgul H, Bourgeois BF, Gauvreau $\mathrm{K}$ and Bergin AM: Electroencephalography in neonatal seizures: Comparison of a reduced and a full 10/20 montage. Pediatr Neurol 32: 155-161, 2005

18. Shah NA and Wusthoff CJ: How to use: Amplitude-integrated EEG (aEEG). Arch Dis Child Educ Pract Ed 100: 75-81, 2015.

19. al Naqeeb N, Edwards AD, Cowan FM and Azzopardi D: Assessment of neonatal encephalopathy by amplitude-integrated electroencephalography. Pediatrics 103: 1263-1271, 1999.

20. Horst HJ, Mud M, Roofthooft MT and Bos AF: Amplitude integrated electroencephalographic activity in infants with congenital heart disease before surgery. Early Hum Dev 86: 759-764, 2010.

21. Fogtmann EP, Plomgaard AM, Greisen G and Gluud C: Prognostic accuracy of electroencephalograms in preterm infants: A systematic review. Pediatrics 139: pii: e20161951, 2017.

22. Latal B, Wohlrab G, Brotschi B, Beck I, Knirsch W and Bernet V: Postoperative amplitude-integrated electroencephalography predicts four-year neurodevelopmental outcome in children with complex congenital heart disease. J Pediatr 178: 55-60.e1, 2016.

23. Drury PP, Gunn AJ, Bennet L, Ganeshalingham A, Finucane K, Buckley D and Beca J: Deep hypothermic circulatory arrest during the arterial switch operation is associated with reduction in cerebral oxygen extraction but no increase in white matter injury. J Thorac Cardiovasc Surg 146: 1327-1333, 2013.

24. Clancy RR, Sharif U, Ichord R, Spray TL, Nicolson S, Tabbutt S, Wernovsky G and Gaynor JW: Electrographic neonatal seizures after infant heart surgery. Epilepsia 46: 84-90, 2005.

25. Bertholdt S, Latal B, Liamlahi R, Pretre R, Scheer I, Goetti R, Dave H, Bernet V, Schmitz A, von Rhein M, et al: Cerebral lesions on magnetic resonance imaging correlate with preoperative neurological status in neonates undergoing cardiopulmonary bypass surgery. Eur J Cardiothorac Surg 45: 625-632, 2014.

26. Gunn JK, Beca J, Penny DJ, Horton SB, d'Udekem YA, Brizard CP, Finucane K, Olischar M, Hunt RW and Shekerdemian LS: Amplitude-integrated electroencephalography and brain injury in infants undergoing Norwood-type operations. Ann Thorac Surg 93: 170-176, 2012.

27. Dimitropoulos A, McQuillen PS, Sethi V, Moosa A, Chau V, Xu D, Brant R, Azakie A, Campbell A, Barkovich AJ, et al: Brain injury and development in newborns with critical congenital heart disease. Neurology 81: 241-248, 2013.

28. Bellinger DC, Wypij D, Rivkin MJ, DeMaso DR, Robertson RL Jr, Dunbar-Masterson C, Rappaport LA, Wernovsky G, Jonas RA and Newburger JW: Adolescents with d-transposition of the great arteries corrected with the arterial switch procedure: Neuropsychological assessment and structural brain imaging. Circulation 124: 1361-1369, 2011.

29. Mulkey SB, Yap VL, Bai S, Ramakrishnaiah RH, Glasier CM, BornemeierRA,Schmitz MLandBhutta AT:Amplitude-integrated EEG in newborns with critical congenital heart disease predicts preoperative brain magnetic resonance imaging findings. Pediatr Neurol 52: 599-605, 2015.

30. Latal B, Kellenberger C, Dimitropoulos A, Hagmann C, Balmer C, Beck I and Bernet V: Can preoperative cranial ultrasound predict early neurodevelopmental outcome in infants with congenital heart disease? Dev Med Child Neurol 57: 639-644, 2015. 
31. Algra SO, Schouten AN, Jansen NJ, van Oeveren W, Haas F, Groenendaal F, Lemmers PM, van Haastert IC, Toet MC and de Vries LS: Perioperative and bedside cerebral monitoring identifies cerebral injury after surgical correction of congenital aortic arch obstruction. Intensive Care Med 41: 2011-2012, 2015.

32. Juan G, Suixin L, Shaoru H, Yunxia S, Yumei L, Yuan R, Chen $\mathrm{C}$ and $\mathrm{Bi} \mathrm{W}$ : Postoperative brain functions in infants with critical congenital heart disease via aEEG. Chin J Thoracic Cardiovascular Surg 34: 581-585, 2018.

33. Gaynor JW, Nicolson SC, Jarvik GP, Wernovsky G, Montenegro LM, Burnham NB, Hartman DM, Louie A, Spray TL and Clancy RR: Increasing duration of deep hypothermic circulatory arrest is associated with an increased incidence of postoperative electroencephalographic seizures. J Thorac Cardiovasc Surg 130: 1278-1286, 2005.
34. von Rhein M, Dimitropoulos A, Valsangiacomo Buechel ER, Landolt MA and Latal B: Risk factors for neurodevelopmental impairments in school-age children after cardiac surgery with full-flow cardiopulmonary bypass. J Thorac Cardiovasc Surg 144: 577-583, 2012.

35. Gaynor JW, Jarvik GP, Bernbaum J, Gerdes M, Wernovsky G, Burnham NB, D'Agostino JA, Zackai E, McDonald-McGinn DM, Nicolson SC, et al: The relationship of postoperative electrographic seizures to neurodevelopmental outcome at 1 year of age after neonatal and infant cardiac surgery. J Thorac Cardiovasc Surg 131: 181-249, 2006. 